- 391

SKIN LESIONS IN LANGERHANS CELL HISTIOCYTOSIS

Eveler $\mathrm{RM}^{1,3}$, de Kraker $\mathrm{J}^{1,4}$, Sillevis Smit JH $\mathrm{H}^{2,4}$, Oranje $\mathrm{AP}^{2,3}$, Hählen $\mathrm{K}^{1,3}$.

Pediatric Oncology' and Dermatology', Sophia Children's Hospital - Rotterdam ${ }^{3}$, Emma Children's Hospital/Academical Medical Center-Amsterdam4, The Netherlands. In considering the clinical features of Langerhans cell histiocytosis ( $\mathrm{LCH}$ ), a proliferation of Langerhans cells, an entire spectrum of findings may be encountered, from minor skin lesions to the diffuse infiltration of abdominal organs associated with continuous fever, weight loss and organ dysfunction. Cutaneous lesions are regarded as a common finding and, not infrequently, the first symptom in $\mathrm{LCH}$. The lesions may have a variety of appearances; most common are brown to red papules which may show vesiculation, ulceration, crusting, or a hemorrhagic appearance. From 1975 to 1993 we reviewed 79 patients with LCH at the Sophia Children's Hospital in Rotterdam and at the Emma Children's Hospital/Academical Medical Center in Amsterdam. Eight patients were diagnosed with pure cutaneous LCH. Four patients were just observed and regressed spontaneously, the other four received a form of treatment. In a tater stage one patient progressed to disseminated LCH with lung involvement. Clinical features at presentation of this patient did not distinguish from the other 7 patients. Of a group of 71 patients with other forms of LCH 28 patients developed skin involvement as part of the disease. The lesions resolved in the patients who were treated with chemotherapy. In those patients who did not receive chemotherapy the skin lesions were either observed or treated locally. We conclude that all patients with LCH apparently localized to the skin should initially be observed, however they need careful staging to assess whether it is part of a disseminated disease.
394

EVALUATION OF N-METHYLHISTAMINE EXCRETION IN URINE OF PATIENTS WITH MASTOCYTOSIS

Dirk Van Gysel ${ }^{2}$, Arnold P Oranje ${ }^{2}$, Ida Vermeijden ${ }^{3}$, J Lijster-de Raadt ${ }^{2}$, Albert $w$ van Toorenenbergen ${ }^{2}$; 'OLV Hospital Aalst, Belgium; 'University Hospital Rotterdam (Sophia) and 'oosterschelde Hospital Goes, the Netherlands.

N-methylhistamine (NMH) excretion was measured in urine of 44 patients with mastocytosis and in urine of 24 age-matched controls, using the double antibody RIA (Kabi-Pharmacia, The Netherlands). Clinical activity of the disease was established in each patient and the patients were further subdivided into 3 groups: mastocytoma (M) urticaria pigmentosa (UP) and diffuse cutacytoma(M), urticaria pig
neous mastocytosis (DCM).

The NMH values in the group of patients with mastocytosis as a whole were significantly higher than in the control group $(p<0.05)$. There was a large overlap in values between the different subtypes. We found a significant difference between the groups of patients with $M$ and DCM $(p<0.01)$, with UP and DCM $(p<0.05)$ and between the groups of patients with active and inactive disease $(p<0.0001)$. We conclude that measurement of NMH excretion in urine can be a useful, but not an absolute tool for diagnosis of mastocytosis and for follow-up of severe disease.
392

IHPORT OF TINEA CAPITIS FROM THE MEDITERRANEAN, AN EPIDEMIC OCCURRENCE IN ROTTERDAM-WEST, THE NETHERLANDS. Y.A.Faber [1], K.Munte [2], C.P. Engelhard [3], M.C.G. Van Praag [1], Arnold p. Oranje [2].

Dept. of Dermatology Sint Franciscus Gasthuis Rotterdam [1], Subdivision of Paediatric Dexmatology, University Hospital Sophia, Rotterdam [2], Department of Infectious Diseases GG \& GD Rotterdam [3], The Netherlands.

In 1993 tinea capitis was diagnosed in 17 index-cases in 2 hospitals in Rotterdam. Six different fungi were determined, of which Microsporum [M] audouinii most frequent ( 6 times). Anthropophilic fungal infections in the Netherlands are especially seen in children from the Mediterranean. Four index-cases caused by M audouinii, Trichophyton [T] mentagrophytes or $\mathrm{T}$ schoenleinii, had minimal 9 infected contacts (familymembers, schoolchildren). The epidemic occurrence could be delineated to a limited area round a school as primary source in Rotterdam-West.

\section{- 393}

JUVENILE HYALINE FIBROMATOSIS - CAUSED BY DISTURBED METABOLISM OF TYPE III COLLAGEN ?

Susanne G. Fang-Kircher, Wolfgang Jurecka*, Fritz Breier*, Konrad Pillwein**

Dept. of Medical Chemistry, Dept. of Dermatology*, Dept. of Pediatrics**, University of Vienna, Vienna, Austria

A defective metabolism of collagen fibrils is the suspected cause of juvenile hyaline fibromatosis (JHF), a rare autosomal recessive disorder characterized by multiple skin tumors, hypertrophic gingivae, flexion contractures and osteolytic bone lesions. All these sites predominantly harbor type-I (TIC) and type-III (TIIIC) collagens. Therefore we analyzed fibroblasts from macroscopically normal, biopsied skin of a $\mathrm{HF}$ patient and a normal individual for markers of TIC and TIIIC metabolism, i.e. propeptides PICP (TIC synthesis), ICTP (TIC degradation) and PIIINP (TIIC metabolism). All experiments were done in triplicates using cell number-matched supernatants. Data are given as mean $\mu \mathrm{g}$ /flask. Both PICP and ICTP were significantly higher in JHF $(24,1$ and 0,516$)$ than in the controls $(12,6$ and 0,324$)$, but their ratio remained unchanged, indicating an accelerated yet balanced TIC metabolism. PIINP was markedly decreased in JHF $(1,05$ vs. 1,65$)$, being reduced by $50 \%$ relative to TIC. The diminished stability of collagen molecules in JHF might be explained by reduced enzymatic removal of these TIIIC-terminal propeptides, which is necessary for regular crosslinking of collagen fibrils. We suggest that the increased TIC turnover, as well as the exessive production of extracellular matrix as observed previously in histological investigations of JHF tissues, are secondary effects of an upset TIIIC metabolism instrumentally involved in the pathogenesis of this disease.

\section{5}

TREATMENT OF PORT-WINE STAINS

author: Chantal M.A.M. van der Horst

Department of Plastic-, Reconstructive- and Handsurgery, Acadenic Medical Centre, Amsterdam, Holland.

Treatment of port-wine stains (PWS) has developed from doing nothing, surgery, cryosurgery, tattoo, dermabrasia, $\mathrm{X}$-ray irradiation, treatment with argon, $\mathrm{CO} 2$, Nd-Yag laser to treatment with pulsed dye laser. With the last one it is for the first time possible to treat children without risk of hypertrophic scarring. Since 1992 patients with PWS in the face, who haven't had any therapy before, are treated with the tunable dye laser. Four age groups $(0-5,6-11,12-17,18-31)$ each consist of 25 patients. Now, 1,5 years later, average treatment of the whole group is 4.6 , which averages 9 sessions. No complete clearance is reached yet. At least a double number of treatments seern to be necessary. Revision of literature shows that 4.6 treatments are mentioned for complete clearance. Final results in treatment are poorly circumscribed.

\section{6}

CUTANEOUS LESIONS AND COMPLICATIONS IN ACUTE MENINGOCOCCAEMIA IN CHILDREN SURVIVING MENINGOCOCCAL SEPTIC SHOCK Spuls Ph.I. ${ }^{1}$, van der Horst C.M.A.M. ${ }^{2}$, Sillevis Smitt J.H. ${ }^{1}$, Derkx H.H.F ${ }^{3}$. Departments of Dermatology ${ }^{1}$, Plastic, Reconstructive and Hand Surgery ${ }^{2}$ and Paediatrics ${ }^{3}$, University of Amsterdam, Academic Medical Centre, The Netherlands

Skin lesions are a presenting sign in fulminant meningococcal septic shock (FMSS) Maculopapular, petechial, ecchymotic lesions and purpura fulminans are related to disseminated intravascular coagulation following endotoxic shock. In order to gain insight into the presence, type, severity, localization, course, complications and follow-up of these skin manifestations in children who survived FMSS, a retrospective analysis of clinical records of 125 children presenting to the Academic Medical Centre with meningococcal infection (of which 52 with FMSS) was performed. Of the 87 children who survived, 23 patients suffered FMSS defined as persistent hypo tension despite volume loading and/or signs of poor end-organ perfusion. Of these, 17 children had severe skin lesions. The lesions tended to be symmetrical and occur red frequently on the upper and lower extremities, thighs and buttocks. The manage ment included intensive nursing care, debridements, necrotomy, skin grafting and amputations. The sequelae consisted of discolorations of the affected skin, multiple atrophic scars and in 4 patients amputations of digits, lower legs and/or arms were required. At present, a prospective study with a multidisciplinary protocol is designed to obtain additional immunological and coagulation parameters on the pathogenesis of the skin lesions and of the effect of treatment, in order to minimize the complications. 